Polymer Journal, Vol. 38, No. 11, pp. 1137-1145 (2006)

(C) 2006 The Society of Polymer Science, Japan

\title{
Thermal and Mechanical Characterization of Electrospun Blends of Poly(lactic acid) and Poly(glycolic acid)
}

\author{
Lisa I. Ramdhanie, ${ }^{1}$ Steven R. Aubuchon, ${ }^{2}$ Eugene D. Boland, ${ }^{1}$ Danielle C. Knapp,${ }^{1}$ \\ Catherine P. BARNES, ${ }^{1}$ David G. Simpson, ${ }^{3}$ Gary E. WNEK,${ }^{4}$ and Gary L. Bowlin ${ }^{1, \dagger}$ \\ ${ }^{1}$ Department of Biomedical Engineering, Virginia Commonwealth University, Richmond, VA 23284-3067 \\ ${ }^{2}$ TA Instruments-Waters LLC, New Castle, DE 19720 \\ ${ }^{3}$ Department of Anatomy and Neurobiology, Virginia Commonwealth University, Richmond, VA 23298-0709 \\ ${ }^{4}$ Department of Chemical Engineering, Case Western Reserve University, Cleveland, OH 44106-7217
}

(Received June 16, 2006; Accepted August 10, 2006; Published October 4, 2006)

\begin{abstract}
Poly(lactic acid) (PLA) and poly(glycolic acid) (PGA) have long been popular polymers in the development of tissue engineering scaffolds due to their biocompatibility, bioabsorbability, and good tensile strength. Electrospinning is an attractive approach for the production of non-woven, nano- to micron-scale fibrous tissue engineering scaffolds of complex geometries. In this study, we characterize electrospun blends of PLA and PGA via scanning electron microscopy, tensile testing, differential scanning calorimetry, and phase contrast microscopy to gain a better understanding of these blended structures for potential use in biomedical applications. [doi:10.1295/polymj.PJ2006062]

KEY WORDS Poly(glycolic acid) / Poly(lactic acid) / Electrospinning / Differential Scanning Calorimetry / Polymer Blend /
\end{abstract}

PLA and PGA are linear aliphatic polyesters often used in biomedical devices (including sutures and tissue engineering scaffolds) particularly because of two properties: biocompatibility and predictable biodegradation via hydrolysis into natural metabolic waste products (glycolic acid and lactic acid which can be metabolized through the Krebs Cycle). Many forms and structures have been utilized in developing medical devices from PLA and PGA and include foams, films, micron-scale fibers, and, more recently, nanoscale fibers by electrospinning. ${ }^{1-6}$ The molecular weight, molecular structure (crystallinity), and morphology play a critical role in the overall mechanical properties and degradation rates of the products fabricated from these polymers.

In electrospinning (Figure 1), a polymer solution (or melt) is held at the end of a capillary tip by its surface tension. An electrostatic charge is applied to the polymer solution in the presence of a strong electric field. Mutual charge repulsion causes a force directly opposite to the surface tension. Relaxation of the induced charge to the free, hemispherical surface of the solution at the tip of the capillary elongates to form a conical shape called a Taylor Cone. As the electric field exceeds a critical value, the electrical repulsion exceeds the surface tension. This results in the emission of a jet stream from the Taylor Cone. The electrical field controls the trajectory of the jet. As the charged jet accelerates and thins in the electric field, the solvent evaporates. Charged polymer fibers ultimately collect on a grounded target. ${ }^{7-10}$

Historically, the majority of electrospinning studies

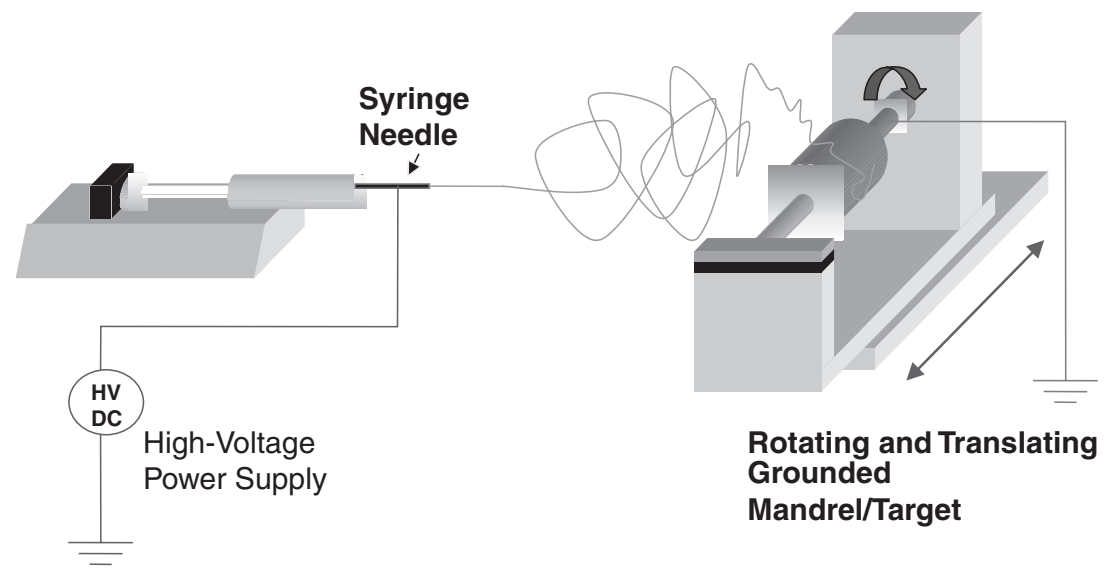

Figure 1. Schematic of an electrospinning apparatus. 
have characterized the non-woven polymer fibrous structures in terms of structural characteristics (e.g., fiber diameter) and mechanical properties (e.g., modulus). The mechanical properties of electrospun PGA have been previously reported. ${ }^{2} \mathrm{Kim}$ and Lee in 2000 published the first study in which differential scanning calorimetry (DSC) was utilized to examine the thermal properties of poly(ethylene terephthalate) (PET), poly(ethylene naphthalate) (PEN), and blends of PET and PEN before and after melt electrospinning. ${ }^{11}$ This study demonstrated that electrospinning resulted in an increase in crystallinity and decreases in glass transition temperature $\left(T_{\mathrm{g}}\right)$ and crystallization peak temperature $\left(T_{\mathrm{c}}\right)$, which they attribute to thermal degradation (reduced molecular weight) after melt spinning and exchange reactions in the melt blends. In 2002, Zong et al. reported the use of DSC to investigate electrospun poly(L-lactic acid) (PLLA) nanofibers electrospun from dimethyl formamide. ${ }^{12}$ The results of this study indicated that the electrospun PLLA nanofibers were non-crystalline but had highly orientated polymer chains and the $T_{\mathrm{g}}$ and $T_{\mathrm{c}}$ were lower than the corresponding values for a cast film. Thus, it was concluded that the electrospinning process significantly impeded the crystallization of PLLA. Another study utilizing DSC and electrospun fibers was published by Shao et al. in 2003 in which they studied poly(vinyl alcohol) (PVA) and silica composite fibers (organic-inorganic hybrids). ${ }^{13}$ This study indicated that electrospun composite fibers gave a new state and special properties of the hybrid that were distinguishable from a gel or film. Finally in 2006, You et al. published a study in which blends of PGA/ PLA (90/10, 70/30, 50/50, 30/70) were electrospun to form ultra-fine fibers where the PLA was removed by selective dissolution to form fibers with threedimensionally interconnected pores. ${ }^{6}$ In this study, DSC results were reported after one cycle of heating to $250^{\circ} \mathrm{C}$. The main result from this study was that for the blended fibers, there were no significant shifts in the melting temperature $\left(T_{\mathrm{m}}\right)$ peaks, indicating that the PLA and PGA are immiscible creating fibers with phase separated structure. This study reported no additional scaffold characterization.

As noted, PGA and PLA are two biodegradable polymers that have a reputable history of use in biomedical devices. The degradation rates of PLA and PGA in biomedical applications are directly related to the microstructure (crystallinity) and morphology of the resultant structure. Another important variable for these and other biomedical polymers is the glass transition temperature which determines the transition from a solid to rubbery state (i.e., modulus), rheological properties, crystallization rate and toughness. In this characterization study, the structure and morphol- ogy of electrospun blends composed of PGA and PLA were investigated and characterized in terms of mechanical and thermal properties. This characterization will provide a better understanding of these structures that is necessary to expand the potential use in biomedical applications.

\section{EXPERIMENTAL}

The following methods were utilized to evaluate the electrospun PLA, PGA, and PLA/PGA blended scaffolds: scanning electron microscopy (SEM), tensile testing, differential scanning calorimetry (DSC), modulated $\mathrm{DSC}^{\circledR}$, and phase contrast microscopy. SEM was used to determine the diameters of the fibers that comprised the scaffolds. Tensile testing was performed to analyze the scaffolds' structural integrity. The DSC and phase contrast microscope were used to corroborate the mechanical properties. The DSC analysis allowed the determination of the percent crystallinity for the blended scaffolds and $T_{\mathrm{g}}, T_{\mathrm{m}}$, and $T_{\mathrm{c}}$. The phase contrast microscope was utilized to indicate whether mechanical weakness might be attributed to phase separation in the electrospinning solutions.

\section{Blending}

For this research, the PGA and PLA obtained from Alkermes, Inc. (Cincinnati, $\mathrm{OH}$ ) were dissolved in 1,1,1,3,3,3,-hexafluoro-2-propanol (HFP, SigmaAldrich Chemical Co.) at a concentration of 0.143 $\mathrm{g} / \mathrm{mL}$ for each. The following blend ratios were used: PGA $100 \%$, PGA 75\%-PLA 25\%, PGA 50\%-PLA $50 \%$, PGA $25 \%$-PLA $75 \%$, and PLA $100 \%$. The polymers were blended together by extracting the required amount of polymer from each stock solution and allowing the polymer to mix on a Fisher Vortex Genie 2 (Fisher Scientific International, Hampton, NH).

\section{Electrospinning}

The solutions were then loaded into a Becton Dickinson $3.0 \mathrm{~mL}$ syringe and placed into a KD Scientific syringe pump set to dispense the solutions at 10 $\mathrm{mL} / \mathrm{h}$. The positive output lead on the Spellman CZE 1000R (Spellman High Voltage Electronics Corp.) was attached to a blunt end 18 gauge needle (approximately $2.5 \mathrm{~cm}$ length) on the syringe. The applied voltage to the needle for charging the polymer solution was $22 \mathrm{kV}$. A grounded stainless steel collecting plate $(7.6 \mathrm{~cm}$ Length $\times 0.5 \mathrm{~cm}$ Width $\times 2.5 \mathrm{~cm}$ Height $)$ was placed $8.0 \mathrm{~cm}$ from the needle tip. To control the orientation of the fibers, the grounded collecting plate was rotated at 360 revolutions per minute (rpm) for mats of randomly oriented fibers and $850 \mathrm{rpm}$ for mats of aligned fibers. 


\section{Scanning Electron Microscopy}

The electrospun fibrous mats were mounted on aluminum stubs, sputter coated with gold, and analyzed using a JEOL JSM-820 JE (JEOL Ltd.) Scanning Electron Microscope (SEM). Each sample was observed using a setting of $15 \mathrm{kV}$ at a magnification of 1000x. The images were digitized and analyzed by UTHSCSA's Image Tool 3.0 (University of Texas Health and Science Center in San Antonio) to determine the average fiber diameter. The averages were based on 60 measurements per micrograph. All measurements were calibrated using the scale on the micrograph as a reference to avoid errors in calculating the magnification of the scanned photos. ${ }^{2,14}$

\section{Tensile Testing}

Using a template, a dog bone shape ${ }^{2,14}$ was cut from each electrospun mat such that longitudinal and transverse samples were obtained from both random and aligned fibrous mats of every composition. Uniaxial tensile testing was performed on a MTS Bionix 200 System (MTS System Corp., Eden Prairie, MN). The uniaxial testing was conducted with the tissue grips extending at a rate of $1.0 \mathrm{~mm} / \mathrm{min}$ and the data acquisition rate was set to $20.0 \mathrm{~Hz}$. The MTS TestWorks ${ }^{\circledR}$ software (Version 4.07 A) was programmed to determine the following material properties: modulus, peak stress, and strain at break.

\section{Differential Scanning Calorimetry}

Pellets of PLA and PGA received from the manufacturer $(5-10 \mathrm{mg}, 0.27 \mathrm{~cm}$ in length and $0.13 \mathrm{~cm}$ in diameter) were analyzed with the TA Instruments Q1000 Differential Scanning Calorimeter (TA Instruments-Waters LLC (TAI), New Castle, DE). A circular section $(5-10 \mathrm{mg}$, diameter $=0.7 \mathrm{~cm}$ and thickness $=0.02 \mathrm{~cm}$ ) was removed from each of the electrospun mats and placed into separate aluminum pans. The temperature profile was programmed and the data analyzed using the Thermal Advantage for Q Series $^{\mathrm{TM}}$ software and Universal Analysis 2000 (TAI). Each sample was heated from $0{ }^{\circ} \mathrm{C}$ to $270{ }^{\circ} \mathrm{C}$ at a rate of $5^{\circ} \mathrm{C} / \mathrm{min}$ and then cooled from $270{ }^{\circ} \mathrm{C}$ to $0^{\circ} \mathrm{C}$ at a rate of $5^{\circ} \mathrm{C} / \mathrm{min}$; each sample was cycled twice for standard DSC and once for modulated $\mathrm{DSC}^{\circledR}$. Both modulated $\left( \pm 0.53^{\circ} \mathrm{C} / 40 \mathrm{~s}\right)$ and standard heating were employed in this study.

The manufacturer provided the percent crystallinity and melt enthalpy for the specific PGA and PLA lot numbers used in this study; these values are given in Table I. In order to estimate the melt enthalpy for a $100 \%$ crystalline sample, the melt enthalpy was divided by the percent crystallinity. Once the thermal curve for each test sample was obtained, the melt enthalpy was determined through integration of the melting
Table I. Manufacturer's reported homopolymer crystallinity

\begin{tabular}{|c|c|c|c|}
\hline \multirow[b]{2}{*}{ Polymer } & \multicolumn{2}{|c|}{ Manufacturer's reported values } & \multirow{2}{*}{$\begin{array}{l}\text { Calculated } \\
\text { melt enthalpy for } \\
100 \% \text { crystallinity }\end{array}$} \\
\hline & $\begin{array}{l}\text { Melt enthalpy } \\
(\mathrm{J} / \mathrm{g})\end{array}$ & $\%$ Crystallinity & \\
\hline PLA & 34.5 & 37.4 & 92.3 \\
\hline PGA & 64.4 & 31.2 & 206 \\
\hline
\end{tabular}

peak. The melt enthalpy calculated for each polymer in each experimental trial was then divided by the theoretical melt enthalpy for $100 \%$ crystallinity as an indication of the percent crystallinity within the polymer.

\section{Phase Contrast Microscopy}

Each of the homopolymer and blended solutions was placed into separate scintillation vials and allowed to coat the inner glass. As a result, a thin film of the blended solution formed on the glass surface. The thinnest, most transparent section, based on visual inspection, in each thin film was placed onto a microscope slide. A Nikon Inverted Microscope TE 300 phase contrast microscope (Nikon Inc., Tokyo, Japan) was then used to evaluate the extent of phase separation within the blends; a magnification of 40x was used.

\section{RESULTS AND DISCUSSION}

\section{Scanning Electron Microscopy}

The SEM micrographs (Figure 2) of electrospun blends of PLA and PGA exhibited a mean fiber diameter range of 0.9 to $1.3 \mu \mathrm{m}$ for aligned fibers and 1.0 to $1.3 \mu \mathrm{m}$ for random fibers; this data is shown graphically in Figure 3. No significant differences exist in the fiber dimensions produced. It should be noted that this fiber diameter size is unachievable by any other conventional fiber production method to date with these polymers. Traditional fiber extrusion and drawing processes can only achieve a 10 to $500 \mu \mathrm{m}$ range. ${ }^{9}$

\section{Tensile Testing}

Tensile testing of the fibrous, non-woven mats demonstrated moduli ranging from 3.4 to $140 \mathrm{MPa}$, peak stress ranging from 2.2 to $11 \mathrm{MPa}$, and percent strain at break ranging from 70 to $330 \%$. The volume fraction of polymer in each electrospun mat is given in Table II. These mechanical properties of the mats can be compared to the mechanical properties of native collagen and elastin, two major structural components in soft tissues such as blood vessels. ${ }^{15}$ Native collagen fibers display the following properties: modulus ranging from 100 to $2900 \mathrm{MPa}$, tensile strength ranging from 5 to $500 \mathrm{MPa}$ and a percent strain at break ranging from 5 to $50 \%$. Native elastin fibers 

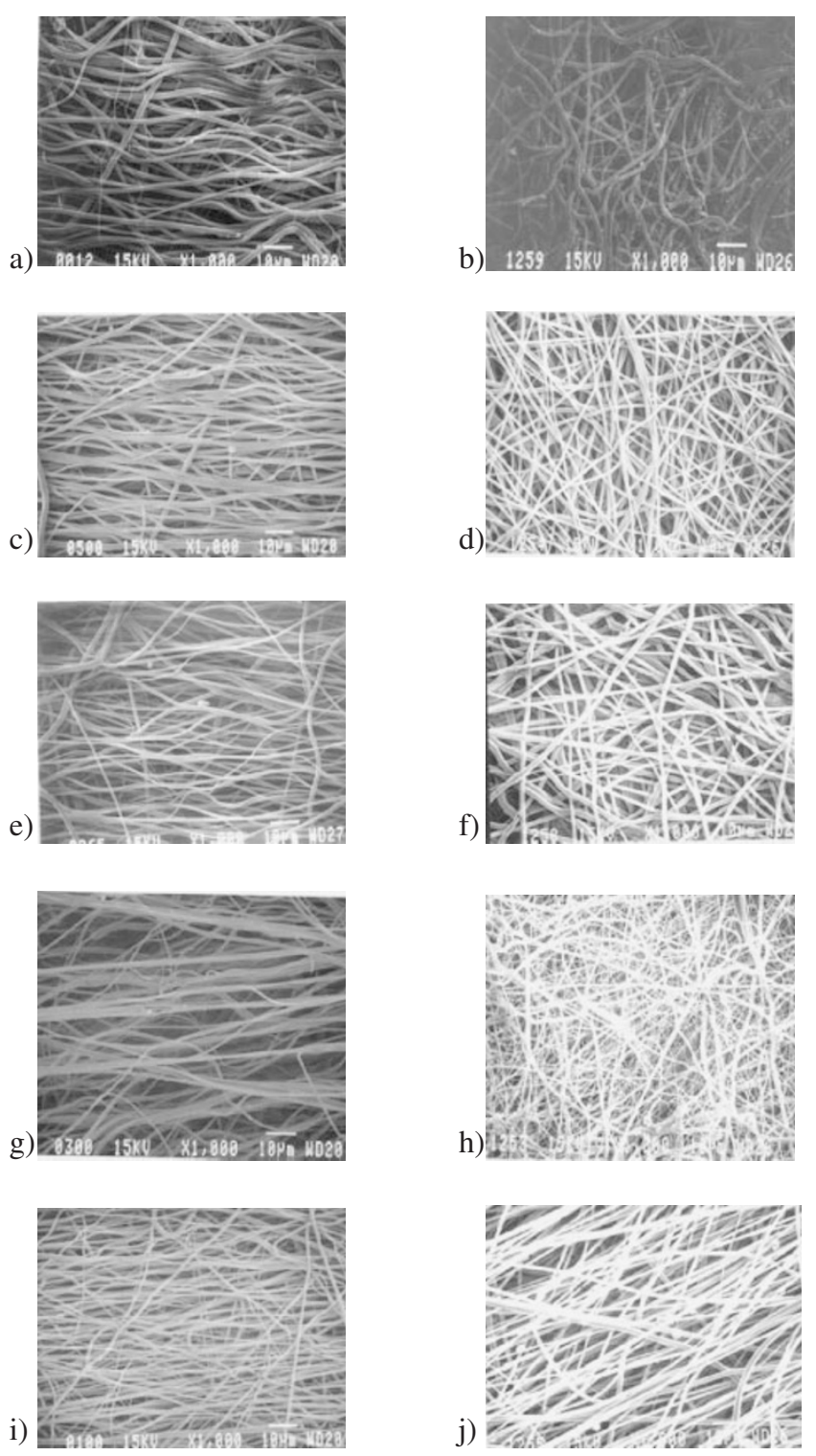

Figure 2. Scanning electron micrographs (magnification 1000x) of a) aligned PLA $100 \%$, b) random PLA $100 \%$, c) aligned PLA 75\%-PGA 25\%,d) random PLA 75\%-PGA 25\%, e) aligned PLA 50\%-PGA 50\%, f) random PLA 50\%-PGA 50\%, g) aligned PLA 25\%-PGA 75\%, h) random PLA 25\%-PGA 75\%, i) aligned PGA $100 \%$, and $\mathrm{j}$ ) random PGA $100 \%$.

exhibit a modulus ranging from 0.3 to $0.6 \mathrm{MPa}$, a tensile strength ranging from 0.36 to $4.4 \mathrm{MPa}$ and a percent strain at break ranging from 100 to $200 \%$. The electrospun blends achieved a modulus between the ranges of collagen and elastin. The blends' percent strain at break range approximates the range for elastin. The peak stress exhibited by electrospun blends of PLA and PGA approximated the lower limit of collagen and the upper limit of elastin.

The mechanical performance for electrospun blends of PLA and PGA were compared with respect to extrinsic properties (architectural design) and intrinsic properties (chemical composition). With respect to architecture, overall, it was found that aligned scaf-

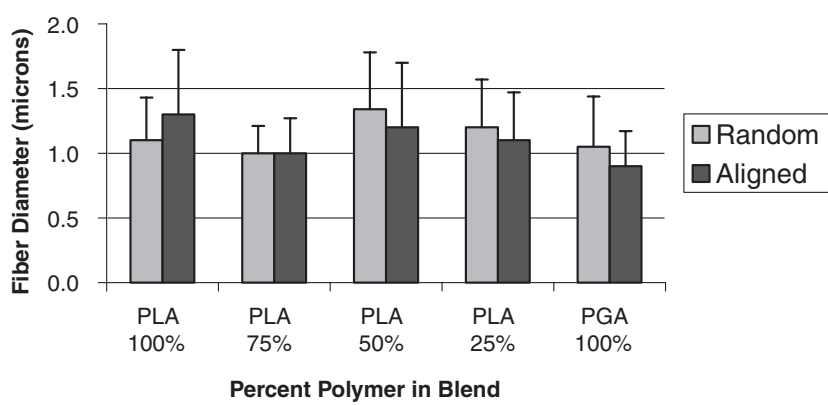

Figure 3. Fiber diameter in the electrospun mats as a function of PLA/PGA solution composition.

Table II. Volume fraction of polymer in electrospun mats

\begin{tabular}{ccc}
\hline Blend & Alignment & Volume fraction \\
\hline \multirow{2}{*}{ PLA } & Aligned & 0.24 \\
& Random & 0.24 \\
PLA 75 & Aligned & 0.27 \\
& Random & 0.28 \\
PLA 50 & Aligned & 0.25 \\
\multirow{2}{*}{ PLA 25 } & Random & 0.26 \\
& Aligned & 0.25 \\
PGA & Random & 0.26 \\
& Aligned & 0.28 \\
\hline
\end{tabular}

folds, when tested longitudinal to the fiber axes, displayed a greater modulus (with PGA concentrations greater than 25\%), higher peak stress, and smaller percent strain at break. Accordingly, aligned scaffolds tested transverse to the fiber axes, exhibited the smallest modulus and the lowest peak stress. Scaffolds with randomly oriented fibers performed similarly to one another when tested longitudinal and transverse to the fiber axes. The effects of composition and orientation on the mechanical properties of the scaffolds are illustrated in Figures 4, 5, and 6.

\section{Differential Scanning Calorimetry}

The observed glass transition temperatures for PGA and PLA as received from the manufacturer, prior to electrospinning, were $42^{\circ} \mathrm{C}$ and $55^{\circ} \mathrm{C}$, respectively, and the observed melting temperatures for PGA and PLA were $173{ }^{\circ} \mathrm{C}$ and $222^{\circ} \mathrm{C}$, respectively. The glass transition temperatures, as measured by modulated DSC $^{\circledR}$, and melting temperatures corresponded well with the material data provided by Alkermes, Inc. The melting temperature was used to determine the percent crystallinity in the material. The electrospinning processing method was able to maintain the homopolymers' integrity during processing, with the exception of PLA 25\%-PGA 75\%. As shown in Table III, there was a significant decrease in crystallinity of the PLA 25\%-PGA 75\% blend, particularly 


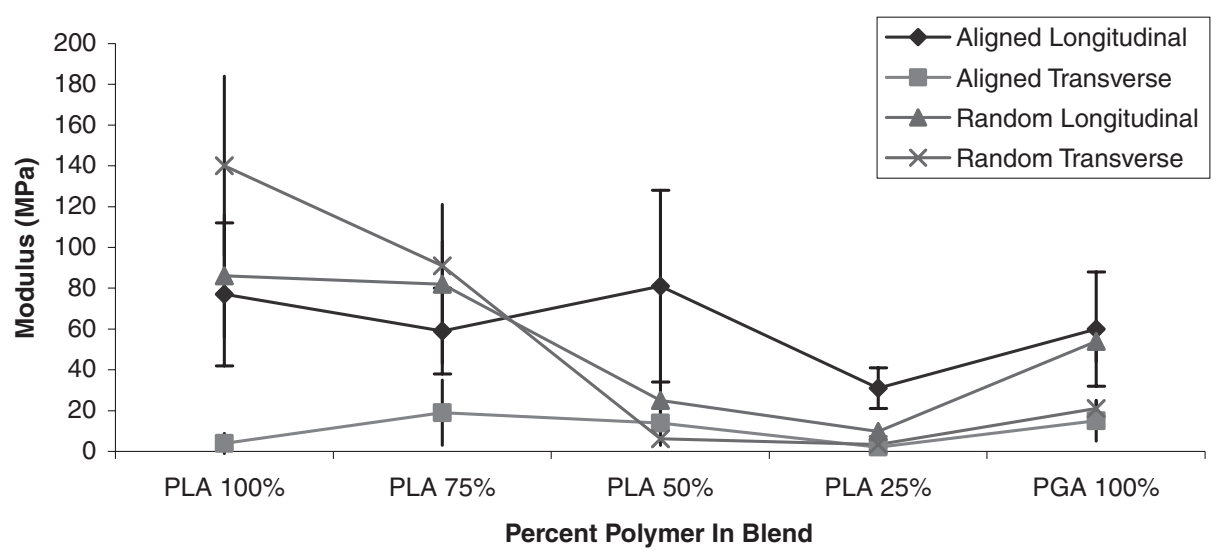

Figure 4. Effects of composition and architecture on modulus.

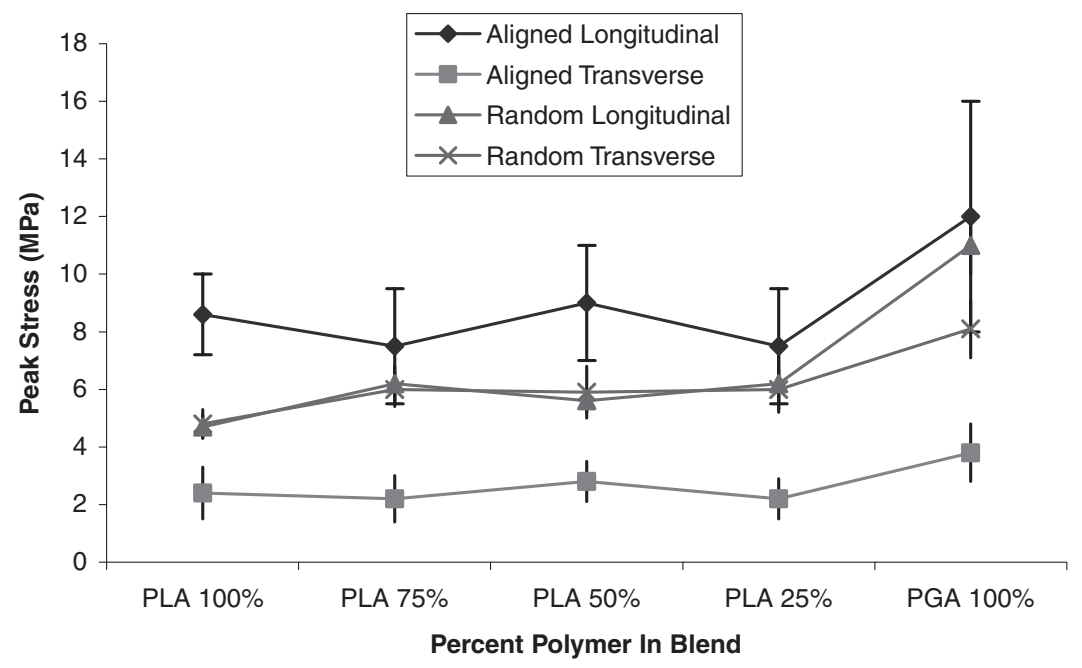

Figure 5. Effects of composition and architecture on peak stress.

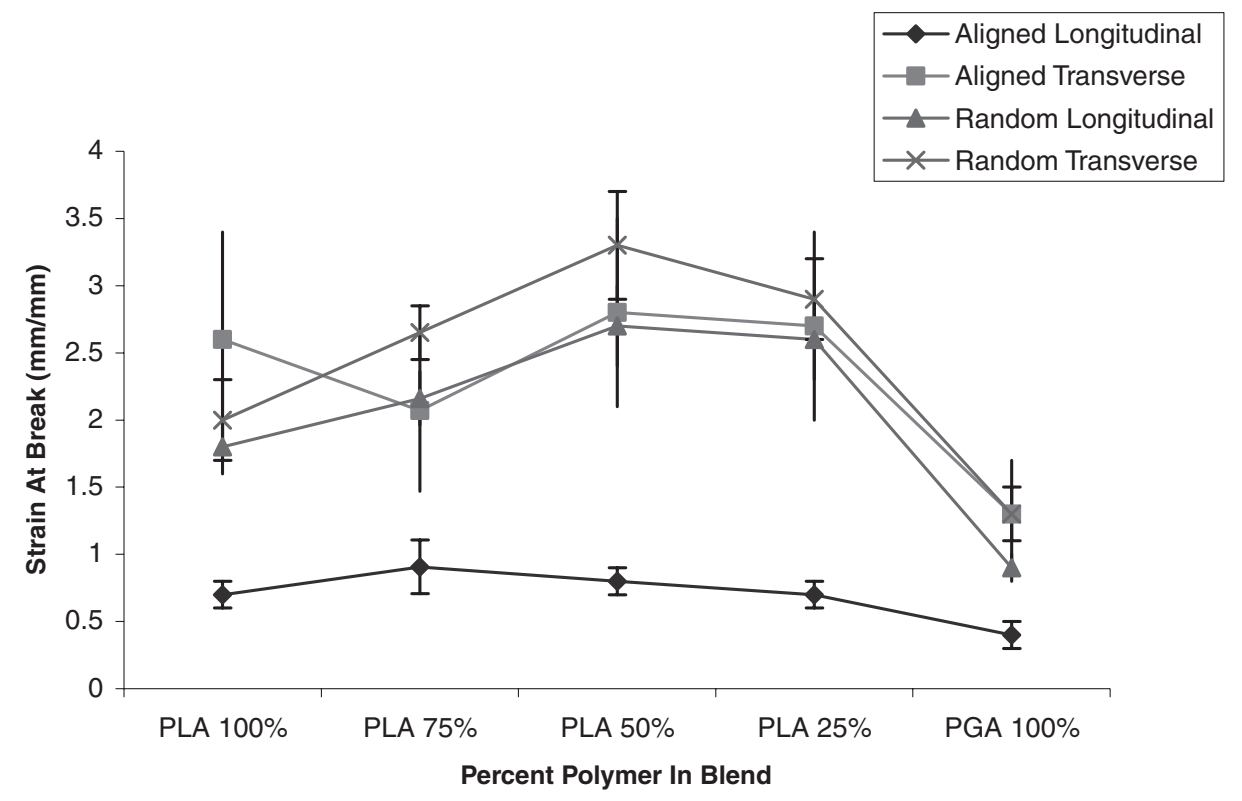

Figure 6. Effects of composition and architecture on strain at break. 
Table III. Standard DSC data

\begin{tabular}{|c|c|c|c|c|c|c|c|}
\hline \multirow{2}{*}{ Blend } & \multirow{2}{*}{ Alignment } & \multirow{2}{*}{ Cycle } & \multicolumn{2}{|c|}{$T_{\mathrm{m}}\left({ }^{\circ} \mathrm{C}\right)$} & \multirow{2}{*}{$\begin{array}{c}T_{\mathrm{g}} \\
\left({ }^{\circ} \mathrm{C}\right)\end{array}$} & \multicolumn{2}{|c|}{$\%$ Crystallinity } \\
\hline & & & PLA & PGA & & PLA & PGA \\
\hline \multirow{2}{*}{ PLA } & \multirow{2}{*}{ Pellet } & 1 & 173 & & 55 & 48 & \\
\hline & & 2 & 173 & & 55 & 49 & \\
\hline \multirow{2}{*}{ PLA } & \multirow{2}{*}{ Aligned } & 1 & 171 & & 32 & 48 & \\
\hline & & 2 & 172 & & 54 & 44 & \\
\hline \multirow{2}{*}{ PLA } & \multirow{2}{*}{ Random } & 1 & 171 & & 31 & 45 & \\
\hline & & 2 & 173 & & 57 & 43 & \\
\hline \multirow{2}{*}{ PLA 75} & \multirow{2}{*}{ Aligned } & 1 & 169 & 219 & 30 & 44 & 39 \\
\hline & & 2 & 173 & 219 & 56 & 47 & 29 \\
\hline \multirow{2}{*}{ PLA 75} & \multirow{2}{*}{ Random } & 1 & 169 & 219 & 29 & 44 & 40 \\
\hline & & 2 & 173 & 219 & 56 & 47 & 32 \\
\hline \multirow{2}{*}{ PLA 75} & \multirow{2}{*}{ Random } & 1 & 169 & 219 & 30 & 41 & 39 \\
\hline & & 2 & 172 & 219 & 56 & 46 & 31 \\
\hline \multirow{2}{*}{ PLA 50} & \multirow{2}{*}{ Aligned } & 1 & 170 & 219 & 29 & 35 & 36 \\
\hline & & 2 & 172 & 219 & 57 & 44 & 28 \\
\hline \multirow{2}{*}{ PLA 50} & \multirow{2}{*}{ Random } & 1 & 172 & 219 & 30 & 39 & 41 \\
\hline & & 2 & 172 & 219 & 53 & 47 & 33 \\
\hline \multirow{2}{*}{ PLA 50} & \multirow{2}{*}{ Random } & 1 & 169 & 219 & 29 & 38 & 40 \\
\hline & & 2 & 171 & 221 & 56 & 47 & 37 \\
\hline \multirow{2}{*}{ PLA 25} & \multirow{2}{*}{ Aligned } & 1 & 170 & 219 & 28 & 9 & 32 \\
\hline & & 2 & 172 & 220 & 41 & 51 & 28 \\
\hline \multirow{2}{*}{ PLA 25} & \multirow{2}{*}{ Random } & 1 & nd & 218 & 29 & - & 38 \\
\hline & & 2 & 172 & 220 & 41 & 48 & 32 \\
\hline PI $A 25$ & & 1 & nd & 219 & 28 & - & 38 \\
\hline PLA 25 & & 2 & 171 & 220 & 41 & 47 & 32 \\
\hline $\mathrm{PG}$ & & 1 & & 222 & 38 & & 37 \\
\hline PGA & Penlet & 2 & & 222 & 42 & & 37 \\
\hline PG & & 1 & & 218 & 29 & & 38 \\
\hline PGA & Al1gned & 2 & & 220 & 39 & & 31 \\
\hline & & 1 & & 219 & 40 & & 35 \\
\hline PGA & Random & 2 & & 221 & 38 & & 31 \\
\hline & & 1 & & 219 & 40 & & 41 \\
\hline TOA & Random & 2 & & 220 & 40 & & 40 \\
\hline
\end{tabular}

nd - not detected.

with respect to the PLA homopolymer. A given set of electrospinning parameters may not maintain the integrity of certain blend ratios. Stitzel reported a similar phenomenon when working with PLA:polycaprolactone (PCL) blends, ranging from $100 \%$ by weight PLA to a $65: 35$ ratio of PLA:PCL. Below this range (e.g., 60:40 PLA:PCL), the spinnability of the polymer blend decreased. Rather than adjusting the parameters in the process, they attempted to characterize the mechanical behavior of polymer blends within a set of processing parameters. ${ }^{16}$

The glass transition temperature for the electrospun mats, however, was not detectable by standard DSC during the first cycle of heating due to the hygroscopic nature of the homopolymers. After dehydration during the first heating cycle, the second heat cycle produced a range of glass transition temperatures from $28^{\circ} \mathrm{C}$ to $57^{\circ} \mathrm{C}$ (See Table III). Since standard DSC was unable to detect the glass transition temperature of the mate-
Table IV. Standard DSC data for the crystallization peak temperature and latent heat of crystallization

\begin{tabular}{|c|c|c|c|c|}
\hline Blend & Alignment & Cycle & $T_{\mathrm{c}}\left({ }^{\circ} \mathrm{C}\right)$ & $\begin{array}{c}\text { Latent heat of } \\
\text { crystallization }(\mathrm{J} / \mathrm{g})\end{array}$ \\
\hline \multirow{2}{*}{ PLA } & \multirow{2}{*}{ Pellet } & 1 & 108 & 41 \\
\hline & & 2 & 107 & 40 \\
\hline \multirow{2}{*}{ PLA } & \multirow{2}{*}{ Aligned } & 1 & 72 & 19 \\
\hline & & 2 & 104 & 32 \\
\hline \multirow{2}{*}{ PLA } & \multirow{2}{*}{ Random } & 1 & 77 & 22 \\
\hline & & 2 & 106 & 35 \\
\hline \multirow{2}{*}{ PLA 75} & \multirow{2}{*}{ Aligned } & 1 & 71 & 16 \\
\hline & & 2 & 107 & 17 \\
\hline \multirow{2}{*}{ PLA 75} & \multirow{2}{*}{ Random } & 1 & 71 & 20 \\
\hline & & 2 & 108 & 22 \\
\hline \multirow{2}{*}{ PLA 75} & \multirow{2}{*}{ Random } & 1 & 71 & 17 \\
\hline & & 2 & 108 & 17 \\
\hline \multirow{2}{*}{ PLA 50} & \multirow{2}{*}{ Aligned } & 1 & 62 & 9 \\
\hline & & 2 & 108 & 11 \\
\hline \multirow{2}{*}{ PLA 50} & \multirow{2}{*}{ Random } & 1 & 57 & 23 \\
\hline & & 2 & 107 & 9 \\
\hline \multirow{2}{*}{ PLA 50} & \multirow{2}{*}{ Random } & 1 & 63 & 21 \\
\hline & & 2 & 106 & 8 \\
\hline \multirow{2}{*}{ PLA 25} & \multirow{2}{*}{ Aligned } & 1 & 58 & 17 \\
\hline & & 2 & 93 & 3 \\
\hline \multirow{2}{*}{ PLA 25} & \multirow{2}{*}{ Random } & 1 & 53 & 6 \\
\hline & & 2 & 92 & 3 \\
\hline \multirow{2}{*}{ PLA 25} & \multirow{2}{*}{ Random } & 1 & 54 & 5 \\
\hline & & 2 & nd & nd \\
\hline \multirow{2}{*}{$\mathrm{PGA}^{\mathrm{a}}$} & \multirow{2}{*}{ Pellet } & 1 & 68 & 8 \\
\hline & & 2 & - & - \\
\hline \multirow{2}{*}{$\mathrm{PGA}^{\mathrm{a}}$} & \multirow{2}{*}{ Aligned } & 1 & 49 & 1.3 \\
\hline & & 2 & - & - \\
\hline \multirow{2}{*}{$\mathrm{PGA}^{\mathrm{a}}$} & \multirow{2}{*}{ Random } & 1 & 49 & 1.3 \\
\hline & & 2 & - & - \\
\hline
\end{tabular}

${ }^{a}$ Values for PGA were obtained from the MDSC data due to being undetected in the standard DSC trials. MDSC and standard DSC data were in agreement for the $T_{\mathrm{c}}$ and latent heat of crystallization data. Only a single cycle was conducted utilizing the modulated DSC. nd - not detected.

rial as used during mechanical testing, modulated DSC $^{\circledR}$ was employed.

Above the glass transition temperature, the polymer chains have mobility. As the temperature is increased, there is a certain temperature, crystallization temperature $\left(T_{\mathrm{c}}\right)$, at which the polymer chains have enough energy to become highly ordered (crystallization or cold crystallization) via an exothermic reaction. This exothermic reaction can be quantified by DSC and is referred to as the latent heat of crystallization. All samples, including the raw material pellets analyzed in this study exhibited a crystallization temperature (Table IV). The PLA and PGA pellets exhibited a $T_{\mathrm{c}}$ of $108^{\circ} \mathrm{C}$ and $68^{\circ} \mathrm{C}$, respectively. Electrospinning of the polymers from HFP into 1.0 to $1.3 \mu \mathrm{m}$ micron diameter fibrous structures lowered the $T_{\mathrm{c}}$ for PLA from 108 to $72-77^{\circ} \mathrm{C}$ and for PGA from 68 to 
$49^{\circ} \mathrm{C}$. As for the blended fibers, increasing amounts of PGA decreased the $T_{\mathrm{c}}$ accordingly with the blend containing only $25 \%$ PLA approaching the $T_{\mathrm{c}}$ of pure electrospun PGA. Similar trends are seen for the latent heat of crystallization. During the second cycle of DSC, all samples approach a $T_{\mathrm{c}}$ value of one of the pure components or a value relative to the blend ratio.

\section{Modulated Differential Scanning Calorimetry ${ }^{\circledR}$}

The melting temperatures determined using modulated DSC results correlated to the standard DSC results as well as to values reported in the literature. Modulated DSC also detected a decrease in the percent crystallinity for the polylactide homopolymer in the PLA 25\%-PGA 75\% blend. The glass transition temperatures of the electrospun mats were shifted below the expected glass transition temperatures for PLA and PGA $\left(55-60^{\circ} \mathrm{C}\right.$ and $45-50^{\circ} \mathrm{C}$, respectively). The glass transition temperature ranges from $30^{\circ} \mathrm{C}$ to $35^{\circ} \mathrm{C}$ (See Table V). The ambient temperature during mechanical testing, uncontrolled, may have been
Table V. $\quad$ Modulated DSC ${ }^{\circledR}$ data

\begin{tabular}{|c|c|c|c|c|c|c|}
\hline \multirow{2}{*}{ Blend } & \multirow{2}{*}{ Alignment } & \multicolumn{2}{|c|}{$T_{\mathrm{m}}\left({ }^{\circ} \mathrm{C}\right)$} & \multirow{2}{*}{$T_{\mathrm{g}}\left({ }^{\circ} \mathrm{C}\right)$} & \multicolumn{2}{|c|}{$\%$ Crystallinity } \\
\hline & & $\overline{\text { PLA }}$ & $\overline{\mathrm{PGA}}$ & & PLA & PGA \\
\hline \multirow{2}{*}{ PLA } & Aligned & 172 & & 31 & 50 & \\
\hline & Random & 171 & & 35 & 45 & \\
\hline \multirow{2}{*}{ PLA 75} & Aligned & 170 & 220 & 32 & 44 & 37 \\
\hline & Random & 170 & 221 & 33 & 44 & 34 \\
\hline \multirow{2}{*}{ PLA 50} & Aligned & 171 & 221 & 32 & 39 & 37 \\
\hline & Random & 169 & 220 & 32 & 36 & 32 \\
\hline \multirow{2}{*}{ PLA 25} & Aligned & 171 & 220 & 32 & 17 & 35 \\
\hline & Random & 169 & 220 & 30 & 5.5 & 34 \\
\hline \multirow{2}{*}{ PGA } & Aligned & & 220 & 31 & & 31 \\
\hline & Random & & 221 & 31 & & 39 \\
\hline
\end{tabular}

below or within this range during some testing. The high surface area to volume ratio (high porosity) results in air as a plasticizer. ${ }^{12}$ In addition, the humidity of the testing conditions could have further softened the materials due to their hygroscopic nature. These variables along with the change in percent crystallinity of PLA 25\%-PGA 75\% could have an affect

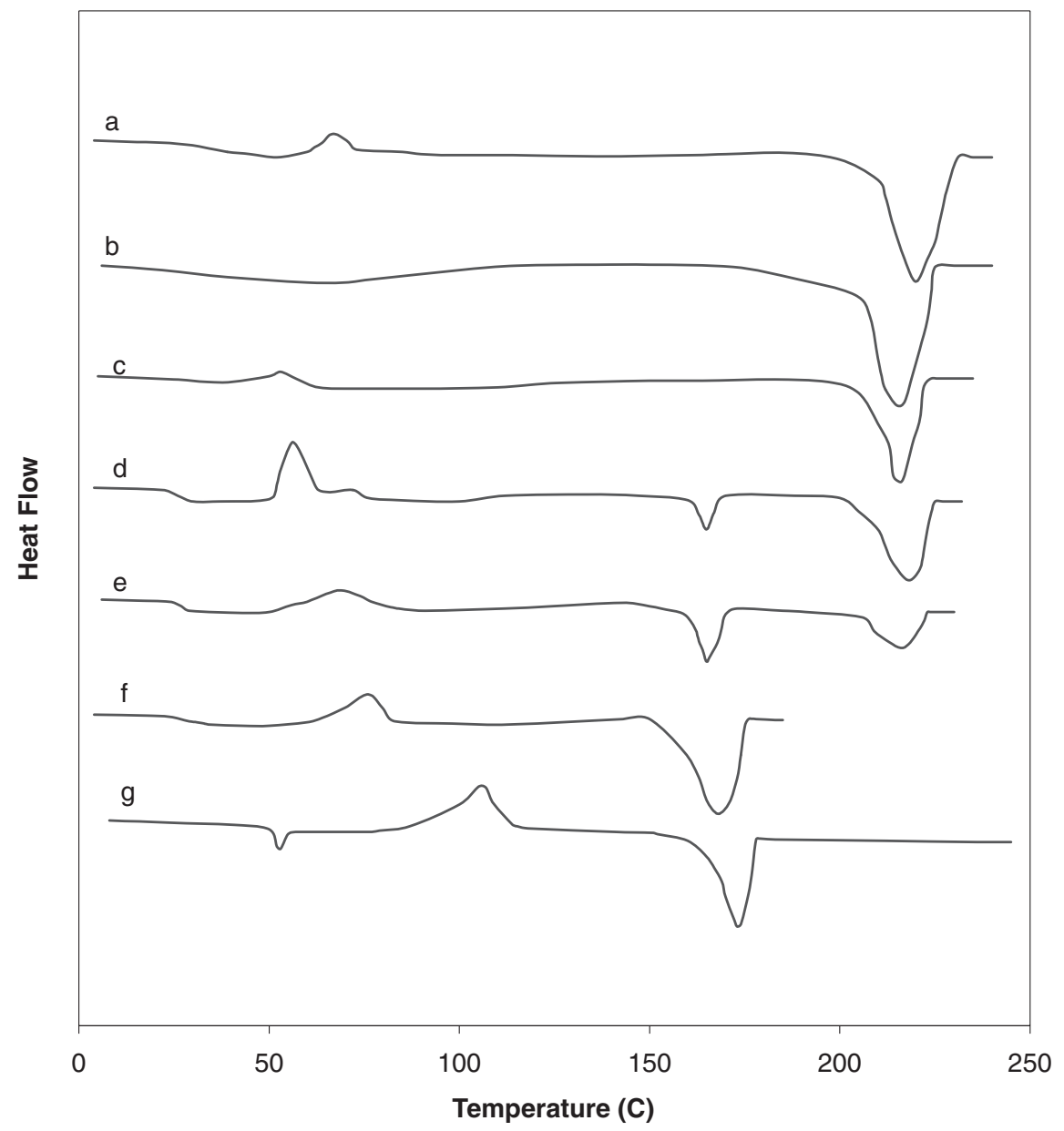

Figure 7. Standard DSC thermograms for a) PGA pellet, b) electrospun PGA, c) electrospun PLA 25\%-PGA 75\%, d) electrospun PLA 50\%-PGA 50\%, e) electrospun PLA 75\%-PGA 25\%, f) electrospun PLA, g) PLA pellet. All electrospun samples presented are random fiber orientation and the first DSC cycle. 

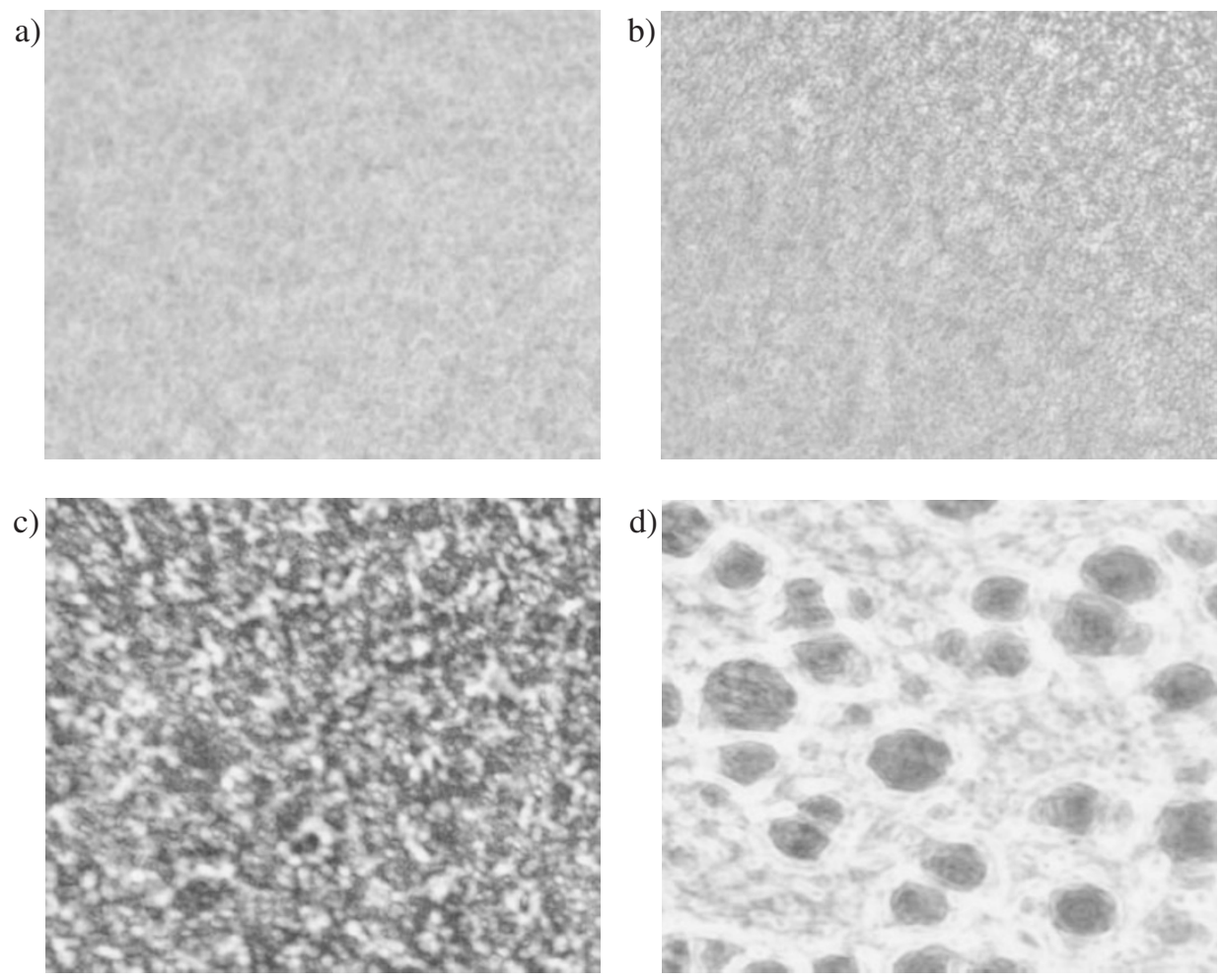

e)

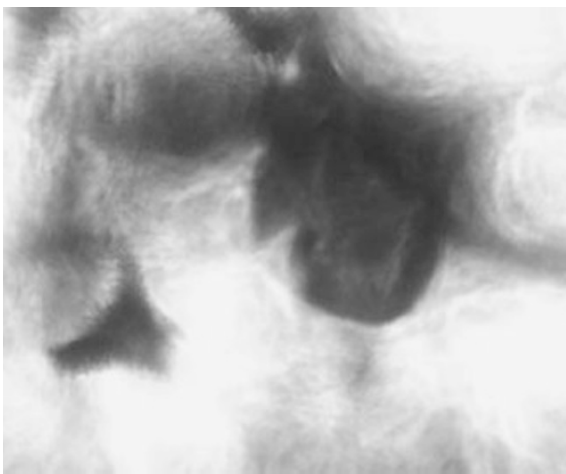

Figure 8. Phase contrast photographs of thin layers of a) PGA, b) PGA 75\%-PLA 25\%, c) PGA 50\%-PLA 50\%, d) PGA 25\%PLA 75\%, and e) PLA; 400x magnification.

on the mechanical performance. Thus, care must be taken in future studies to control ambient conditions during all aspects of processing and testing of these materials.

Finally, the miscibility of a polymer blend can be determined by analyzing the $T_{\mathrm{m}}$. In a polymer blend, if one of the components is crystalline, then its crystalline structure will be hindered by a miscible component in the blend. Thus, the $T_{\mathrm{m}}$ and crystallinity should significantly decrease with increasing addition of the blended component. It is clear from the DSC data (Figure 7) obtained that there was no significant shift in the $T_{\mathrm{m}}$ for the blends versus the pure polymers, indicating that the PGA and PLA in HFP are immiscible and that the fibers are phase separated structures. These results support the results presented by You et $a .^{6}$

\section{Phase Contrast Microscopy}

The phase contrast microscope was used to determine the extent of phase separation within a thin film cast from the evaporation of the electrospinning solutions. Large regions of PLA and PGA, instead of a uniform distribution of the two polymers, would explain outliers, large standard deviations, and unpredicted trends in mechanical behavior.

The phase contrast microscope analysis (Figure 8) indicated a uniform distribution of at least two phases in all compositions. The properties and behavior observed are indeed the behavior and properties of the blended polymers. Qualitatively, a higher degree of phase separation was observed for polymers with a high concentration of PLA, including PLA 50\%PGA 50\% and PLA 75\%-PGA 25\%. Although it is yet unclear how strong the correlation is between 
solution properties and the properties of an electrospun mat, the observed phase separation does provide a suggestion that inter-phasial weakness may occur.

\section{CONCLUSIONS}

Overall, the morphology and mechanical properties of electrospun blends of PLA and PGA indicate that these materials are promising and may be utilized in soft tissue engineering. The fiber morphology of the electrospun blends, which approaches that of the native ECM, could be more conducive to cell attachment, infiltration, and proliferation. Furthermore, the mechanical properties of the electrospun blends approximate the lower range of mechanical properties of native soft tissues, such as blood vessel tensile properties (with respect to collagen and elastin mechanical properties). The DSC and phase contrast microscopy results have served as possible explanations for the mechanical behavior of the materials. The temperature in which testing was conducted may have varied around the electrospun material's glass transition temperature, as detected by DSC. In addition, humidity was variable during testing. These variables may have plasticized the blends to different degrees on different days. It is unknown what effect phase separation prior to electrospinning has on the fiber arrangement during the electrospinning process. Therefore, it could have been possible to have domains of each polymer within the electrospun mat, versus a uniform distribution of polymer fibers.

Acknowledgment. The authors would like to thank the Whitaker Foundation (RG-98-0465) for the support of this research. We would also like to thank Ms. Judy Williamson for her assistance obtaining the SEM micrographs.

\section{REFERENCES}

1. W. H. Wong and D. Mooney, in "Synthetic Biodegradable Polymer Scaffolds,” A. Atala, D. Mooney, J. P. Vacanti, and R. Langer, Ed., Birkhauser, Boston, MA. 1997, p. 51.

2. E. D. Boland, G. E. Wnek, D. G. Simpson, K. J. Pawlowski, and G. L. Bowlin, J. Macromol. Sci., Part A: Pure Appl. Chem., 38, 1231 (2001).

3. E. D. Boland, T. A. Telemeco, D. G. Simpson, G. E. Wnek, and G. L. Bowlin, J. Biomed. Mater. Res., Part B, 71, 144 (2004).

4. J. D. Stitzel, K. J. Pawlowski, G. E. Wnek, D. G. Simpson, and G. L. Bowlin, J. Biomater. Appl., 15, 1 (2001).

5. K. Kim, M. Yu, X. Zong, J. Chiu, D. Fang, Y.-S. Seo, B. S. Hsiao, B. Chu, and M. Hadjiargyrou, Biomaterials, 24, 4977 (2003).

6. Y. You, J. H. Youk, S. W. Lee, B.-M. Min, S. J. Lee, and W. H. Park, Mater. Lett., 60, 757 (2006).

7. J. M. Deitzel, J. Kleinmeyer, D. Harris, and N. C. B. Tan, Polymer, 42, 261 (2001).

8. J. Doshi and D. H. Reneker, J. Electrostatics, 35, 151 (1995).

9. Y. M. Shin, M. M. Hohman, M. P. Brenner, and G. C. Rutledge, Appl. Phys. Lett., 78, 1149 (2001).

10. J. M. Deitzel, J. D. Kleinmeyer, J. K. Hirvonen, and N. C. B. Tan, Polymer, 42, 8163 (2001).

11. J. S. Kim and D. S. Lee, Polym. J., 32, 616 (2000).

12. X. Zong, K. Kim, D. Fang, S. Ran, B. S. Hsiao, and B. Chu, Polymer, 43, 4403 (2002).

13. C. Shao, H.-Y. Kim, J. Gong, B. Ding, D.-R. Lee, and S.-J. Park, Mater. Lett., 57, 1579 (2003).

14. E. D. Boland, B. D. Coleman, C. P. Barnes, D. G. Simpson, G. E. Wnek, and G. L. Bowlin, Acta Biomater., 1, 115 (2005).

15. W. M. Abbott and R. P. Cambria, in "Biological and synthetic vascular prostheses,” J. C. Stanley, Ed., Grune and Stratton, New York, NY., 1982, p. 189.

16. J. D. Stitzel, Master's Thesis in Biomedical Engineering, Virginia Commonwealth University, Richmond, VA., 2000. 